\title{
On a contraction property of Bernoulli canonical processes
}

by

\section{Witold BEDNORZ and Rafał MARTYNEK}

\author{
Presented by Stanisław KWAPIEN
}

Summary. We give several results concerning suprema of canonical processes. The main theorem concerns a contraction property of Bernoulli canonical processes which generalizes the one proved by Talagrand (1993). It states that for independent Rademacher random variables $\left(\varepsilon_{i}\right)_{i \geq 1}$ we can compare $\mathbf{E} \sup _{t \in T} \sum_{i \geq 1} \varphi_{i}(t) \varepsilon_{i}$ with $\mathbf{E}_{\sup _{t \in T}} \sum_{i=1}^{\infty} t_{i} \varepsilon_{i}$, where the function $\varphi=\left(\varphi_{i}\right)_{i \geq 1}: T \rightarrow \ell^{2}, T \subset \ell^{2}$, satisfies certain conditions. Originally, it was assumed that each $\varphi_{i}$ is a contraction. We relax this assumption to comparability of Gaussian parts of increments: for all $s, t \in T$ and $p \geq 0$,

$$
\inf _{\left|I^{c}\right| \leq C p} \sum_{i \in I}\left|\varphi_{i}(t)-\varphi_{i}(s)\right|^{2} \leq C^{2} \inf _{\left|I^{c}\right| \leq p} \sum_{i \in I}\left|t_{i}-s_{i}\right|^{2},
$$

where $C \geq 1$ is an absolute constant and $I \subset \mathbb{N}, I^{c}=\mathbb{N} \backslash I$.

1. Introduction and notation. Throughout this paper we will use the following notation. For a set $A$ the number of elements in $A$ will be denoted as $|A|$. If $t=\left(t_{i}\right)_{i \geq 1}$ is a sequence of real numbers and $p \geq 1$ then $\|t\|_{p}=\left(\sum_{i=1}^{\infty}\left|t_{i}\right|^{p}\right)^{1 / p}$, and $\ell^{p}$ is the space of all sequences $t$ with $\|t\|_{p}<\infty$. If $S, T \subset \ell^{p}$ then $S+T=\{s+t: s \in S, t \in T\}$. For a random variable $\xi$ and $p>0$ we put $\|\xi\|_{p}=\left(\mathbf{E}|\xi|^{p}\right)^{1 / p}$. If $\left(\xi_{i}\right)_{i \geq 1}$ is a sequence of independent, identically distributed random variables such that $\mathbf{E} \xi_{i}=0, \mathbf{E} \xi_{i}^{2}=1$ and $t=\left(t_{i}\right)_{i \geq 1} \in \ell^{2}$ then the random variable

$$
X_{t}=\sum_{i=1}^{\infty} t_{i} \xi_{i}
$$

2010 Mathematics Subject Classification: 60G15, 60G17, 60G50.

Key words and phrases: Bernoulli process, Bernoulli comparison, canonical processes, chaining methods, weak and strong moments.

Received 27 March 2017; revised 22 June 2019.

Published online 23 July 2019. 
is well-defined. For each $T \subset \ell^{2}$ with $0 \in T$ the process $X_{T}=\left(X_{t}\right)_{t \in T}$ is called canonical. The above series converges in $\|\cdot\|_{2}$, i.e.

$$
\lim _{n \rightarrow \infty}\left\|\sum_{i=1}^{n} t_{i} \xi_{i}-X_{t}\right\|_{2}=0
$$

Clearly,

$$
\left\|X_{t}-X_{s}\right\|_{2}=\|t-s\|_{2} \quad \text { for } s, t \in T \text {. }
$$

REMARK 1. The almost sure convergence in (1) might be guaranteed also when the independence assumption on $\xi_{i}$ 's is skipped. In that case we may consider a finite-dimensional version of $(1)$, where $T \subset \mathbb{R}^{d}$. The most studied example is when $\xi_{i}$ 's have log-concave tails, i.e. $\mathbf{P}\left(\left|\xi_{i}\right|>t\right)=\exp \left(-N_{i}(t)\right)$ for $N_{i}:[0, \infty] \rightarrow[0, \infty]$ convex, and may be dependent.

We want to distinguish two types of canonical processes which will be of special interest. If $\left(\xi_{i}\right)=\left(\varepsilon_{i}\right)$ and $\mathbf{P}\left(\varepsilon_{i}=1\right)=\mathbf{P}\left(\varepsilon_{i}=-1\right)=1 / 2$ then the process $X_{T}$ is called canonical Bernoulli and denoted by $B_{T}=$ $\left(B_{t}\right)_{t \in T}$. This class of processes is important for various applications, e.g. to infinitely divisible processes [18] and empirical processes (see [19] for a comprehensive study). If $\left(\xi_{i}\right)=\left(g_{i}\right)$ and $g_{i}$ are normally $\mathcal{N}(0,1)$ distributed then the process $X_{T}$ is called canonical Gaussian and denoted by $G_{T}=$ $\left(G_{t}\right)_{t \in T}$. In fact, canonical Gaussian processes can be seen as a motivation to study canonical processes in general, the reason being the KarhunenLoève representation of separable Gaussian processes by means of canonical Gaussian processes (see e.g. [12, Corollary 5.3.4]).

The main object studied will be suprema of canonical processes. For any set $T$ and a stochastic process $\left(X_{t}\right)_{t \in T}$ we define

$$
S_{X}(T)=\sup _{F \subset T} \mathbf{E} \sup _{t \in F} X_{t}
$$

where $F$ runs through all finite subsets of $T$. Usually, by considering a separable modification of $X_{t}, t \in T$, it is possible to guarantee that $\sup _{t \in T} X_{t}$ is a well-defined random variable (for the definition of a separable version of a process and a discussion of measurability of suprema in the general setting of not necessarily separable Banach spaces see [11, Ch. 2]). In this case $S_{X}(T)$ coincides with the usual expectation of the supremum of $X_{t}$, i.e.

$$
S_{X}(T)=\mathbf{E} \sup _{t \in T} X_{t}
$$

Let us finish this section with a few important technicalities which will be helpful in dealing with canonical processes. We have $S_{X}(T)=S_{X}(T-t)$, where $T-t=\{s-t: s \in T\}$, so we may always require that $0 \in T$. Moreover, $S_{X}(T)=S_{X}(\operatorname{Conv} T)$ and $S_{X}(T)=S_{X}(\operatorname{cl} T)$, where Conv $T$ is the convex hull of $T$ and $\operatorname{cl} T$ is the closure of $T$ in $\ell^{2}$. 
We follow the convention that numerical constants denoted by the same letter may vary from line to line.

2. Suprema of canonical processes via chaining. First, we recall the basics of the chaining approach to upper bounds for stochastic processes. Let $(T, d)$ be a separable metric space, $t_{0} \in T$ a fixed element and $X_{t}, t \in T$, a process such that $\left\|X_{s}-X_{t}\right\|_{2} \leq d(t, s)$ for $t, s \in T$, so that it is separable. For each countable dense $D \subset T$ it is true that $\mathbf{E} \sup _{t \in D} X_{t}=S_{X}(T)$.

We say that a sequence $\mathcal{A}=\left(\mathcal{A}_{n}\right)_{n \geq 0}$ of partitions of $T$ is admissible if $\mathcal{A}_{0}=\{T\}$ and $\left|\mathcal{A}_{n}\right| \leq N_{n}=2^{2^{n}}$ for $n \geq 1$ and these partitions are nested, i.e. for any $A \in \mathcal{A}_{n}, n \geq 1$, there is $B \in \mathcal{A}_{n-1}$ such that $A \subset B$. For $t \in T$ we denote by $A_{n}(t)$ the unique element of the partition $\mathcal{A}_{n}$ which contains $t$.

A sequence $\pi=\left(\pi_{n}\right)_{n \geq 0}$ of mappings $\pi_{n}: T \rightarrow T$ is said to be adapted to the partitions $\left(\mathcal{A}_{n}\right)_{n \geq 0}$ if $\pi_{n}(t)=\pi_{n}(s)$ for $s, t \in A \in \mathcal{A}_{n}, n \geq 0$, and $\pi_{0}(t)=t_{0}$ for $t \in T$ (the common value of $\pi_{n}$ on $A \in \mathcal{A}_{n}$ will be denoted by $\left.\pi_{n}(A)\right)$. Let $T_{n}=\left\{\pi_{n}(t): t \in T\right\}$ and $D=\bigcup_{n} T_{n}$.

We say that $\pi$ is regular if

$$
\lim _{n \rightarrow \infty} d\left(t, \pi_{n}(t)\right)=0 \quad \text { for each } t \in T .
$$

For regular $\pi$ the set $D$ is dense in $T$. We define

$$
\gamma_{X}(\pi)=\sup _{t \in T} \sum_{n=1}^{\infty}\left\|X_{\pi_{n}(t)}-X_{\pi_{n-1}(t)}\right\|_{2^{n}}, \quad \gamma_{X}(T)=\inf \gamma_{X}(\pi)
$$

where the infimum is taken over all admissible sequences $\left(\mathcal{A}_{n}\right)_{n \geq 0}$ of partitions of $T$ and regular sequences $\pi_{n}, n \geq 0$, of mappings $T \rightarrow T$ adapted to $\left(\mathcal{A}_{n}\right)_{n \geq 0}$. Furthermore, for $t \in T$ and each $m>1$ we can write the following chain representation:

$$
X_{\pi_{m}(t)}=X_{\pi_{0}(t)}+\sum_{n=1}^{m}\left(X_{\pi_{n}(t)}-X_{\pi_{n-1}(t)}\right) .
$$

Therefore for each $t \in D$ we have

$$
X_{t} \leq X_{t_{0}}+\sum_{n=1}^{\infty}\left|X_{\pi_{n}(t)}-X_{\pi_{n-1}(t)}\right| .
$$

$\gamma_{X}(T)$ should be compared with Talagrand's functional $\gamma_{2}(T, d)$ (see [19, Definition 2.2.19] or the formulation due to Latała and Mendelson (see [9], [13]), where it was proved that under suitable regularity assumptions, $S_{X}(T) \leq$ $K \gamma_{X}(T)$, where $K$ is a universal constant.

Let us give a short argument for a similar upper bound with an improved constant. 
THEOREM 1. Under the above assumption on $(T, d), t_{0} \in T$ and a process $\left(X_{t}\right)_{t \in T}$ we have

$$
S_{X}(T) \leq \mathbf{E} X_{t_{0}}+3 \gamma_{X}(T) .
$$

Proof. Let $\left(\mathcal{A}_{n}\right)_{n \geq 0}$ be any admissible sequence of partitions of $T$ and $\pi=\left(\pi_{n}\right)_{n \geq 0}$ a regular and sequence of mappings of $T$ adapted to $\left(\mathcal{A}_{n}\right)_{n \geq 0}$. For any $A \in \mathcal{A}_{n}$ and $n \geq 1$ we denote by $A^{\prime}$ the unique element of $\mathcal{A}_{n-1}$ which contains $A$.

In what follows we will use the fact that $a \leq b\left(1+(a / b-1)_{+}\right)$for any $a, b>0$. For each $t \in D$ we get

$$
\begin{aligned}
& X_{t}-X_{t_{0}} \leq \sum_{n=1}^{\infty}\left|X_{\pi_{n}(t)}-X_{\pi_{n-1}(t)}\right| \\
& \leq \sum_{n=1}^{\infty} 2\left\|X_{\pi_{n}(t)}-X_{\pi_{n-1}(t)}\right\|_{2^{n}}\left(1+\left(\frac{\left|X_{\pi_{n}(t)}-X_{\pi_{n-1}(t)}\right|}{2\left\|X_{\pi_{n}(t)}-X_{\pi_{n-1}(t)}\right\|_{2^{n}}}-1\right)_{+}\right) \\
& \leq\left(\sum_{n=1}^{\infty} 2\left\|X_{\pi_{n}(t)}-X_{\pi_{n-1}}(t)\right\|_{2^{n}}\right)\left(1+\sum_{n=1}^{\infty}\left(\frac{\left|X_{\pi_{n}(t)}-X_{\pi_{n-1}(t)}\right|}{2\left\|X_{\pi_{n}(t)}-X_{\pi_{n-1}(t)}\right\|_{2^{n}}}-1\right)_{+}\right) .
\end{aligned}
$$

The last inequality holds since $\sum_{n=1}^{\infty} b_{n}\left(1+c_{n}\right) \leq\left(\sum_{n=1}^{\infty} b_{n}\right)\left(1+\sum_{n=1}^{\infty} c_{n}\right)$ for any sequences $\left(b_{n}\right),\left(c_{n}\right)$ of nonnegative real numbers.

Hence,

$$
\begin{aligned}
\sup _{t \in D} X_{t}-X_{t_{0}} \leq & \left(\sup _{t \in T} \sum_{n=1}^{\infty} 2\left\|X_{\pi_{n}(t)}-X_{\pi_{n-1}(t)}\right\|_{2^{n}}\right) \\
& \cdot\left(1+\sum_{n=1}^{\infty} \sup _{t \in T}\left(\frac{\left|X_{\pi_{n}(t)}-X_{\pi_{n-1}(t)}\right|}{2\left\|X_{\pi_{n}(t)}-X_{\pi_{n-1}(t)}\right\|}-1\right)_{+}\right) \\
\leq & 2 \gamma_{X}(\pi)\left(1+\sum_{n=1}^{\infty} \sum_{A \in \mathcal{A}_{n}}\left(\frac{\left|X_{\pi_{n}(A)}-X_{\pi_{n-1}\left(A^{\prime}\right)}\right|}{2\left\|X_{\pi_{n}(A)}-X_{\pi_{n-1}\left(A^{\prime}\right)}\right\|_{2^{n}}}-1\right)_{+}\right) .
\end{aligned}
$$

We easily see that if $t \in A \in \mathcal{A}_{n}$ then

$$
X_{\pi_{n}(t)}-X_{\pi_{n-1}(t)}=X_{\pi_{n}(A)}-X_{\pi_{n-1}\left(A^{\prime}\right)} .
$$

Therefore,

$$
\begin{aligned}
& \mathbf{E} \sup _{t \in D} X_{t} \\
& \leq \mathbf{E} X_{t_{0}}+2 \gamma_{X}(\pi)\left(1+\sum_{n=1}^{\infty} \sum_{A \in \mathcal{A}_{n}} \mathbf{E}\left(\frac{\left|X_{\pi_{n}(A)}-X_{\pi_{n-1}\left(A^{\prime}\right)}\right|}{2\left\|X_{\pi_{n}(A)}-X_{\pi_{n-1}\left(A^{\prime}\right)}\right\|_{2^{n}}}-1\right)_{+}\right) .
\end{aligned}
$$

Now, we show that for any nonnegative random variable $\theta$ and $p>2$,

$$
\mathbf{E}\left(\frac{\theta}{2\|\theta\|_{p}}-1\right)_{+} \leq \frac{1}{2} \cdot \frac{1}{p \cdot 2^{p}}
$$


Obviously, it is enough to prove that if $\theta \geq 0$ and $\mathbf{E} \theta^{p} \leq 1$ then $\mathbf{E}(\theta / 2-1)_{+} \leq$ $\frac{1}{2} \cdot \frac{1}{p \cdot 2^{p}}$. Indeed, if $\theta$ is as above and $\xi=\mathbf{E}(\theta \mid \mathcal{G})$ where $\mathcal{G}$ is the $\sigma$-field generated by the single event $C=\{\theta \geq 2\}$ then $\mathbf{E}(\xi / 2-1)_{+}=\mathbf{E}(\theta / 2-1)_{+}$and by Jensen's inequality $\mathbf{E} \xi^{p} \leq \mathbf{E} \theta^{p} \leq 1$. Observe that for the random variable $\xi$ we have $\xi=x \mathbb{1}_{C}$ for some $x>2$ and $C$ with $\mathbf{P}(C) \leq 1 / x^{p}$. Hence

$$
\mathbf{E}\left(\frac{\theta}{2}-1\right)_{+}=\mathbf{E}(\xi) \leq \max _{x>2} \frac{1}{x^{p}}\left(\frac{x}{2}-1\right)=\left(1-\frac{1}{p}\right)^{p-1} \frac{1}{p \cdot 2^{p}} \leq \frac{1}{2} \cdot \frac{1}{p \cdot 2^{p}} .
$$

If we apply the above inequality for $p=2^{n}$ for each $n \geq 1$, the inequality (4) yields

$$
\mathbf{E} \sup _{t \in D} X_{t}-\mathbf{E} X_{t_{0}} \leq 2 \gamma_{X}(\pi)\left(1+\frac{1}{2} \cdot \sum_{k=1}^{\infty} \frac{N_{k}}{2^{k} 2^{2^{k}}}\right) \leq 3 \gamma_{X}(\pi) .
$$

Hence taking the infimum over all admissible partitions together with regular and adapted sequences $\pi$ we conclude the proof.

The same proof gives the estimate

$$
\mathbf{E} \sup _{t \in T}\left|X_{t}\right| \leq \mathbf{E}\left|X_{t_{0}}\right|+3 \gamma_{X}(T)
$$

Lemma 1. Let $X=\left(X_{t}\right), t \in \ell^{2}$, be a canonical process and $C_{1}$ a constant such that

$$
\left\|X_{t}\right\|_{2^{n+1}} \leq C_{1}\left\|X_{t}\right\|_{2^{n}}, \quad \forall t \in \ell^{2}, n \geq 0 .
$$

Then, for all $T_{1}, T_{2} \subset \ell^{2}$.

$$
\gamma_{X}\left(T_{1}+T_{2}\right) \leq C_{1}\left(\gamma_{X}\left(T_{1}\right)+\gamma_{X}\left(T_{2}\right)\right) .
$$

For canonical Bernoulli and canonical Gaussian processes the above inequality holds with $C_{1}=\sqrt{3}$.

Proof. For $i=1,2$ let $\left(\mathcal{A}_{n}^{i}\right)$ be an admisible sequence of partitions of $T_{i}$, together with an adapted and regular sequence $\pi^{i}=\left(\pi_{n}^{i}\right)$ of mappings of $T_{i}$. We have to construct an admissible sequence of partitions for $T_{1}+T_{2}$ with an associated sequence of mappings. For that purpose for each $t \in T_{1}+T_{2}$ fix $t^{1} \in T_{1}$ and $t^{2} \in T_{2}$ such that $t=t^{1}+t^{2}$. For $A^{1} \subset T_{1}$ and $A^{2} \subset T_{2}$ define

$$
A^{1} * A^{2}=\left\{t \in T_{1}+T_{2}: t^{1} \in A^{1}, t^{2} \in A^{2}\right\} .
$$

Define $\mathcal{B}_{n}$ in the following way: $\mathcal{B}_{0}=\left\{T_{1}+T_{2}\right\}$ and for $n \geq 0$ let $\mathcal{B}_{n+1}$ consist of all sets $A^{1} * A^{2}$, where $A^{1} \in \mathcal{A}_{n}^{1}$ and $A^{2} \in \mathcal{A}_{n}^{2}$. It is easy to see that $\left(\mathcal{B}_{n}\right)$ is an admissible sequence of partitions of $T_{1}+T_{2}$. Indeed, $\left|\mathcal{B}_{n+1}\right| \leq N_{n} \cdot N_{n} \leq N_{n+1}$, the sequence is clearly nested and for $t=t^{1}+t^{2}$ we have $B_{n}(t)=A_{n}\left(t^{1}\right) * A_{n}^{\prime}\left(t^{2}\right)$.

Now define a sequence $\pi=\left(\pi_{n}\right)_{n \geq 0}$ by $\pi_{0}(t)=0$ and $\pi_{n+1}(t)=\pi_{n}^{1}\left(t^{1}\right)+$ $\pi_{n}^{2}\left(t^{2}\right)$ for $t \in T^{1}+T^{2}$. Obviously the sequence $\left(\pi_{n}\right)$ is regular and adapted 
to the partition $\left(\mathcal{B}_{n}\right)$. Furthermore,

$$
\left\|t-\pi_{n+1}(t)\right\|_{2} \leq\left\|t^{1}-\pi_{n}^{1}\left(t^{1}\right)\right\|_{2}+\left\|t^{2}-\pi_{n}^{2}\left(t^{2}\right)\right\|_{2},
$$

so $\lim _{n \rightarrow \infty}\left\|t-\pi_{n+1}(t)\right\|_{2}=0$. In this way we guarantee the regularity condition (2) for the sequence of mappings $\pi_{n}$ adapted to the partition $\mathcal{B}_{n}$.

We need to show that for fixed $t \in T_{1}+T_{2}$,

$$
\sum_{n=1}^{\infty}\left\|X_{\pi_{n}(t)}-X_{\pi_{n-1}(t)}\right\|_{2^{n}} \leq C_{1}\left(\gamma_{X}\left(T_{1}\right)+\gamma_{X}\left(T_{2}\right)\right) .
$$

By the above construction and the triangle inequality we get

$\left\|X_{\pi_{n+1}(t)}-X_{\pi_{n}(t)}\right\|_{2^{n+1}} \leq\left\|X_{\pi_{n}^{1}\left(t^{1}\right)}-X_{\pi_{n-1}^{1}\left(t^{1}\right)}\right\|_{2^{n+1}}+\left\|X_{\pi_{n}^{2}\left(t^{2}\right)}-X_{\pi_{n-1}^{2}\left(t^{2}\right)}\right\|_{2^{n+1}}$, so by (5),

$\left\|X_{\pi_{n+1}(t)}-X_{\pi_{n}(t)}\right\|_{2^{n}} \leq C_{1}\left(\left\|X_{\pi_{n}^{1}\left(t^{1}\right)}-X_{\pi_{n-1}^{1}\left(t^{1}\right)}\right\|_{2^{n}}+\left\|X_{\pi_{n}^{2}\left(t^{2}\right)}-X_{\pi_{n-1}^{2}\left(t^{2}\right)}\right\|_{2^{n}}\right)$

and we sum over $n \geq 1$ to obtain (7). That (5) holds with constant $\sqrt{3}$ for both canonical Bernoulli and Gaussian processes is a result known as hypercontractivity (cf. [1], [7, Chapter 3.4], [4, Chapter 13]), which states that for $1<q<p<\infty$ we have $\left\|B_{t}\right\|_{p} \leq \sqrt{\frac{p-1}{q-1}}\left\|B_{t}\right\|_{q}$, and similarly for $G_{t}$.

Let us summarize the available information about the processes for which a full characterization of the supremum (i.e. lower and upper bounds) can be provided with the use of $\gamma_{X}(T)$. The seminal result of Fernique and Talagrand known as the Majorizing Measure Theorem (see [3], [16] or [19] for a modern formulation) is equivalent to the statement that $S_{G}(T)$ is comparable with $\gamma_{G}(T)$ up to a numerical constant. In [17] it was proved that $S_{X}(T)$ is comparable with a quantity which, in a sense, is equivalent to $\gamma_{X}(T)$ for a canonical process generated by $\xi$ 's which are symmetric and satisfy $\mathbf{P}(|\xi|>t)=\exp \left(-c_{p} t^{p}\right)$ for a fixed $p \in[1,2]$. A similar result holds for $p>2$, yet it is only possible to show that there exists a set $T^{\prime} \subset \ell^{2}$ (which may significantly differ from $T$ ) such that $S_{X}(T)$ is comparable with $\gamma_{X}\left(T^{\prime}\right)$ up to a numerical constant. Note that the limiting case when $p \rightarrow \infty$ is the case of canonical Bernoulli processes.

Later, the idea of [17] was slightly generalized by R. Latała [8] to canonical processes generated by $\xi$ with log-concave tails, yet under specific regularity assumptions. Finally, in [10] it was proved that it suffices to assume only certain conditions on the moment growth of $\xi$. Unfortunately, this result still does not apply to Bernoulli processes.

The question of characterizing $S_{B}(T)$ was a long-standing problem posed by M. Talagrand and known as the Bernoulli conjecture. It was finally settled in [2]. In order to explain this result we need to provide a family of distances relating to canonical Bernoulli processes which follow from some properties 
of Bernoulli-type random variables. We have (see [5], [14] and [6] for the formulation below), for any $p \in \mathbb{N}, p \geq 1$,

$$
\left\|B_{t}\right\|_{p} \leq \sum_{i=1}^{p}\left|t_{i}^{*}\right|+\sqrt{p}\left(\sum_{i>p}\left|t_{i}^{*}\right|^{2}\right)^{1 / 2} \leq 4\left\|B_{t}\right\|_{p}
$$

where $\left(t_{i}^{*}\right)_{i \geq 1}$ is the rearrangement of $\left(t_{i}\right)_{i \geq 1}$ such that $\left|t_{1}^{*}\right| \geq\left|t_{2}^{*}\right| \geq \cdots$. Equivalently, we can express this relation as

$$
\frac{1}{4} \inf _{t=t^{1}+t^{2}}\left(\left\|t^{1}\right\|_{1}+\left\|G_{t^{2}}\right\|_{p}\right) \leq\left\|B_{t}\right\|_{p} \leq \inf _{t=t^{1}+t^{2}}\left(\left\|t^{1}\right\|_{1}+\left\|G_{t^{2}}\right\|_{p}\right)
$$

for $p \geq 2$. This motivates the following interpretation. If we denote by $I \subset \mathbb{N}$ some index set, we can think of (8) as a decomposition of the norm $\left\|B_{t}\right\|_{p}$ into the $\ell^{1}$ part

$$
\sum_{i=1}^{p}\left|t_{i}^{*}\right|=\sup _{\left|I^{c}\right| \leq p} \sum_{i \in I^{c}}\left|t_{i}\right|
$$

and the Gaussian part

$$
\sqrt{p}\left(\sum_{i>p}\left|t_{i}^{*}\right|^{2}\right)^{1 / 2}=\sqrt{p} \inf _{\left|I^{c}\right| \leq p}\left(\sum_{i \in I}\left|t_{i}\right|^{2}\right)^{1 / 2} .
$$

In fact, a characterization similar to (8) can be formulated for a broad class of processes, namely processes with log-concave distributions. In particular, in [9] there is a characterization of $\left\|X_{t}-X_{s}\right\|_{p}$ for canonical processes based on one-unconditional log-concave random variables.

As already mentioned, the characterization of $S_{B}(T)$ was known as the Bernoulli conjecture and was finally proved in [2]. It states that similarly to (8), $S_{B}(T)$ can be decomposed into the Gaussian and $\ell^{1}$ parts. More precisely, there must exist a decomposition of $T$ into $T_{1}, T_{2} \subset \ell^{2}$ such that $T_{1}+T_{2} \supset T$ and moreover $S_{B}(T)$ dominates up to a universal constant both $\sup _{t \in T_{1}}\|t\|_{1}$ and $S_{G}\left(T_{2}\right)$. Usually such a decomposition is formulated in terms of existence of a mapping $\pi: T \rightarrow \ell^{2}$ which defines $T_{1}=\{t-\pi(t): t \in T\}$ and $T_{2}=\{\pi(t): t \in T\}$. Recall that we can always assume that $0 \in T$ and $\pi(0)=0$.

We now prove that the Bernoulli Theorem of [2] implies that there must exist a subset $T^{\prime} \subset \ell^{2}$ such that $\gamma_{B}\left(T^{\prime}\right)$ is comparable to $S_{B}(T)$. The idea of the proof works also for other classes of canonical processes for which we can characterize $S_{X}(T)$ in terms of increments (see Remark 3 below).

THEOREM 2. There exists a function $\pi: T \rightarrow \ell^{2}$ such that

$$
K^{-1}\left(\gamma_{B}\left(T_{1}+T_{2}\right)\right) \leq S_{B}(T) \leq K\left(\gamma_{B}\left(T_{1}+T_{2}\right)\right)
$$

where $K$ is a universal constant, $T_{1}=\{t-\pi(t): t \in T\}$ and $T_{2}=\{\pi(t)$ : $t \in T\}$. 
Proof. By the main result of [2] we get the existence of $\pi: T \rightarrow \ell^{2}$ and consequently the existence of a decomposition into countable sets $T_{1}, T_{2} \subset \ell^{2}$ such that $T \subset T_{1}+T_{2}$ and

$$
S_{B}(T) \geq K^{-1}\left(\sup _{t \in T_{1}}\|t\|_{1}+S_{G}\left(T_{2}\right)\right),
$$

where $K$ is a universal constant. By the famous Fernique-Talagrand majorizing measure bound ([16], [15], 3]) we know that $S_{G}(T)$ is comparable with $\gamma_{G}\left(T_{2}\right)$. To be precise, we know that $S_{G}(T) \geq C \gamma_{2}(T)$, where $\gamma_{2}(T)$ is Talagrand's $\gamma_{2}$ functional given by

$$
\inf \sup _{t \in T_{2}} \sum_{n=0}^{\infty} 2^{n / 2} \Delta_{2}\left(A_{n}(t)\right),
$$

where the infimum runs over all admissible sequences of partitions of $T_{2}$ and $\Delta_{2}$ denotes the diameter of a set in $\ell^{2}$ norm. Obviously, we associate with any admissible partition $\left(\mathcal{A}_{n}\right)_{n \geq 0}$ a sequence $\left(\pi_{n}\right)$ by choosing $\pi_{n}(t)$ to be any point in $A_{n}(t)$. Notice that the diameters of the sets $A_{n}(t)$ converge uniformly to 0 so (5) is satisfied. To conclude that $\gamma_{2}\left(T_{2}\right) \geq C \gamma_{G}\left(T_{2}\right)$ we just estimate the $2^{n}$ th norm of a Gaussian random variable by the 2 nd norm. Now, let $g$ be a standard normal variable independent of $B_{t}, t \in T$. Observe that for any $p \geq 1$,

$$
\frac{\sqrt{2}}{\sqrt{\pi}}\left\|B_{t}-B_{s}\right\|_{p}=\mathbf{E}|g|\left\|B_{t}-B_{s}\right\|_{p} \leq\left\|G_{t}-G_{s}\right\|_{p}
$$

so we can conclude $\frac{\sqrt{\pi}}{\sqrt{2}} \gamma_{G}\left(T_{2}\right) \geq \gamma_{B}\left(T_{2}\right)$.

The next goal is to show that $\sup _{t \in T_{1}}\|t\|_{1} \geq C \gamma_{B}\left(T_{1}\right)$. To this end we consider a dense countable subset $S_{1}$ of $T_{1}$. We will start by constructing an admissible sequence of partititions and the associated sequence $\left(\pi_{n}\right)$ for $S_{1}$ and then we will extend the construction to the whole $T_{1}$. We choose an admissible sequence $\left(\mathcal{A}_{n}\left(S_{1}\right)\right)_{n \geq 0}$ of partitions such that for $n>0, \mathcal{A}_{n}\left(S_{1}\right)$ consists of $N_{n}-1$ single points and one addititonal set that contains all the remaining points. Clearly this sequence of partitions is nested. Fix $t_{0}$ in $S_{1}$. Define

$$
\pi_{n}(t)= \begin{cases}t & \text { if } A_{n}(t)=\{t\} \\ t_{0} & \text { otherwise }\end{cases}
$$

Obviously, this sequence of partitions together with the sequence $\left(\pi_{n}\right)$ defined above satisfy (2). If for some $m, t \in A_{m}(t)=\{t\}$ but $A_{m-1}(t) \neq\{t\}$, then

$$
\sum_{n=1}^{\infty}\left\|B_{\pi_{n}(t)}-B_{\pi_{n-1}(t)}\right\|_{2^{n}}=\left\|B_{t}-B_{t_{0}}\right\|_{2^{m}} \leq\left\|t-t_{0}\right\|_{1} .
$$


Therefore, by the triangle inequality,

$$
\sup _{t \in S_{1}} \sum_{n=1}^{\infty}\left\|B_{\pi_{n}(t)}-B_{\pi_{n-1}(t)}\right\|_{2^{n}} \leq 2 \sup _{t \in S_{1}}\|t\|_{1} .
$$

In this way we have proved that $\sup _{t \in S_{1}}\|t\|_{1} \geq \frac{1}{2} \gamma_{B}\left(S_{1}\right)$.

Now, we provide a procedure that allows us to extend the construction of the partition and the sequence $\left(\pi_{n}\right)$ to the whole $T_{1}$. Recall that the partition element $\mathcal{A}_{n}\left(S_{1}\right)$ consists of $N_{n}-1$ singletons which we will denote by $S^{n}$. Of course, $S^{1} \subset S^{2} \subset \cdots$. Furthermore, $S_{1}$ is countable, so we can refer to some fixed order on $S_{1}$. To construct the partition $\mathcal{A}_{n}\left(T_{1}\right)$ we will proceed by induction. For $n=0$ we simply put $\mathcal{A}_{0}\left(T_{1}\right)=\left\{T_{1}\right\}, \pi_{0}(t)=t_{0} \in S_{1} \cap T_{1}$, since $S_{1} \cap T_{1} \neq \emptyset$. Suppose we have constructed $\mathcal{A}_{n-1}\left(T_{1}\right), \pi_{n-1}(t), t \in T_{1}$, $n \geq 2$. Consider $A \in \mathcal{A}_{n-1}\left(T_{1}\right)$. For $t \in A$ we define $\pi_{n}(t)$ as the element $s$ of $S^{n-1}$ which minimizes $\|t-s\|_{2}$. In the case of multiple minimizers we choose the smallest one in the assumed order. This mapping defines a partition of the set $A$ into no more than $N_{n-1}-1$ elements. We repeat this procedure for the remaining elements of $\mathcal{A}_{n-1}\left(T_{1}\right)$ to obtain $\mathcal{A}_{n}\left(T_{1}\right)$, which is nested in the obvious way. Moreover, its cardinality does not exceed $N_{n-1} \cdot N_{n-1}=N_{n}$. Finally, the construction of $\pi_{n}(t)$ guarantees the regularity condition (5). This finishes the construction.

By Lemma 1 we obtain

$$
S_{B}(T) \geq K^{-1}\left(\gamma_{B}\left(T_{1}\right)+\gamma_{B}\left(T_{2}\right)\right) \geq\left(K C_{1}\right)^{-1} \gamma_{B}\left(T_{1}+T_{2}\right) .
$$

On the other hand, we have a trivial upper bound

$$
S_{B}(T) \leq S_{B}\left(T_{1}\right)+S_{B}\left(T_{2}\right)=S_{B}\left(T_{1}+T_{2}\right) \leq 4 \gamma_{B}\left(T_{1}+T_{2}\right),
$$

by Theorem 1 .

REMARK 2. The natural lower bound in the above theorem would of course be $\gamma_{B}(T)$ rather than $\gamma_{B}\left(T_{1}+T_{2}\right)$. However, it is not necessarily true that $\gamma_{B}$ is monotone in the sense that $\gamma_{B}(T) \leq \gamma_{B}\left(T_{1}+T_{2}\right)$ despite the fact that $T \subset T_{1}+T_{2}$. The problem is that $\pi_{n}(T) \subset T_{1}+T_{2}$ but we cannot easily rearrange $\pi_{n}$ so that $\pi_{n}(T) \subset T$. Obviously, we could reformulate our definition in a way that $\pi_{n}: T \rightarrow \ell^{2}$. In this setting $\gamma_{B}$ is monotone but values of $\pi$ may still stay outside $T$. It is a non-trivial question whether it is possible to improve the choice of the decomposition map $\pi$ on the set $T$ so that $\pi(T) \subset T$.

Let us also observe that for $\mathbf{P}\left(\left|\xi_{i}\right|>t\right)=\exp \left(-c_{p} t^{p}\right), p \geq 2$, we could give a similar proof, since for any $p$ we have Talagrand's [19] characterization of $S_{X}(T)$.

REMARK 3. For the class of canonical processes based on independent symmetric $\xi_{i}$ such that $\mathbf{P}\left(\left|\xi_{i}\right|>t\right)=\exp \left(-c_{p} t^{p}\right), p \geq 2, S_{X}(T)$ is comparable 
with $\gamma_{X}\left(T_{1}+T_{2}\right)$ up to a constant for some $T_{1}+T_{2} \subset \ell^{2}$ that contains $T$. The role of $T_{2}$ can again be associated with comparing the process $X$ with the Gaussian process, whereas $T_{1} \subset \ell^{p^{*}}$ for $p^{*}=\frac{p}{p-1}$.

In general, we conjecture that the same is true for canonical processes based on log-concave random variables.

CONJECTURE 1. If $\left(\xi_{i}\right)_{i \geq 1}$ is a sequence of independent log-concave random variables with mean 0 and variance 1 then there exist $\pi: T \rightarrow \ell^{2}$ such that if $T_{1}=\left\{t-\pi(t) \in \ell^{2}: t \in T\right\}$ and $T_{2}=\left\{\pi(t) \in \ell^{2}: t \in T\right\}$, then

$$
K^{-1}\left(\gamma_{X}\left(T_{1}+T_{2}\right)\right) \leq S_{X}(T) \leq K\left(\gamma_{X}\left(T_{1}+T_{2}\right)\right),
$$

where $K$ is a universal constant.

3. Contractions of canonical Bernoulli processes. Suppose we have a map $\varphi: T \rightarrow \ell^{2}$. The main question we treat in this paper is under what assumptions on $X_{t}, T$ and $\varphi$ we can show that $S_{X}(\varphi(T))$ is bounded by $S_{X}(T)$ up to a numerical constant. In particular we are interested in the case of canonical Bernoulli processes.

Let us start with classical results concerning comparison of Gaussian processes. It is well-known that if $G_{t}$ and $G_{t}^{\prime}, t \in T$, are centered Gaussian processes and $\mathbf{E}\left|G_{t}-G_{s}\right|^{2} \leq \mathbf{E}\left|G_{t}^{\prime}-G_{s}^{\prime}\right|^{2}$, then for each finite subset $F \subset T$,

$$
\mathbf{E} \sup _{t \in F} G_{t} \leq \mathbf{E} \sup _{t \in F} G_{t}^{\prime}
$$

This is a consequence of Slepian's Lemma ([11, Corollary 3.14] provides the proof with constant 2; the proof with the best possible constant 1 is in 3 , Corollary 2.1.3]). Note also that by the Majorizing Measure Theorem the result can be generalized to the case where we compare a centered Gaussian process with a centered process for which we only require subgaussianity (see [11, Theorem 12.16]).

We start with a discussion of possible extensions of this result. It is natural to ask for other cases when similar comparison results hold. From Theorem 1 it can be easily deduced that if we can compare moments then we can compare $\gamma$-type upper bounds.

Corollary 1. Suppose that $\left(X_{t}\right)_{t \in T}$ is a canonical process and $\varphi: T \rightarrow \ell^{2}$. If there exists a universal constant $C$ such that for each $n \geq 1$,

$$
\left\|X_{\varphi(t)}-X_{\varphi(s)}\right\|_{2^{n}} \leq C\left\|X_{t}-X_{s}\right\|_{2^{n}},
$$

then $S_{X}(\varphi(T)) \leq 3 C \gamma_{X}(T)$.

Proof. Clearly, by Theorem 1, $S_{X}(\varphi(T)) \leq 4 \gamma_{X}(\varphi(T)) \leq 3 C \gamma_{X}(T)$.

This means that if we could show that $S_{X}(t) \geq K^{-1} \gamma_{X}(T)$, then by Corollary 1 we would get $S_{X}(\varphi(T)) \leq 3 C K S_{X}(T)$. Unfortunately, in general, there is no proof that $\gamma_{X}(T)$ is comparable with $S_{X}(T)$. On the other hand, 
as discussed before, there are cases where the idea works. In particular, we could use Corollary 1 in order to recover the Gaussian comparison result with some absolute constant. However, in the Gaussian setting, one can simply refer to 11), rewriting it in the following way:

if $\varphi: T \rightarrow \ell^{2}$ satisfies $\|\varphi(t)-\varphi(s)\|_{2} \leq\|t-s\|_{2}$, then $S_{G}(\varphi(T)) \leq S_{G}(T)$.

We now move to the case of canonical Bernoulli processes. The only known comparison result is [18, Theorem 2.1] (or [11, Theorem 4.12]). It states that if $\varphi=\left(\varphi_{i}\right)_{i \geq 1}: T \rightarrow \ell^{2}$, where $\varphi_{i}: \mathbb{R} \rightarrow \mathbb{R}$ are contractions, then $S_{B}(T)$ dominates $S_{B}(\varphi(T))$ with constant 1:

$$
\text { if }\left|\varphi_{i}(x)-\varphi_{i}(y)\right| \leq|x-y| \text { for } i \geq 1 \text {, then } S_{B}(\varphi(T)) \leq S_{B}(T) \text {. }
$$

Note that if we are interested only in comparison up to a numerical constant (not necessarily 1) then the requirement of coordinate contractions is too demanding. However, it is known that the result analogous to (11), where we assume that $\varphi: \ell^{2} \rightarrow \ell^{2}$ is a Lipschitz contraction, does not hold for Bernoulli processes. Therefore some additional assumptions on $\varphi$ or $T$ are required.

As we show in this paper, comparison for canonical Bernoulli processes depends on a suitable family of distances already present in (8). The following comparison result is a straightforward consequence of Theorem 2 .

Corollary 2. Suppose that $\varphi: T \rightarrow \ell^{2}$ can be extended to $T_{1}+T_{2}$ in such a way that for any $p \geq 1$,

$$
\left\|B_{\varphi(t)}-B_{\varphi(s)}\right\|_{p} \leq\left\|B_{t}-B_{s}\right\|_{p} \quad \text { for all } s, t \in T_{1}+T_{2} .
$$

Then $S_{B}(\varphi(T)) \leq K S_{B}(T)$, where $K$ is a universal constant.

Proof. Clearly, by Theorem 1 we have $S_{B}(\varphi(T)) \leq 3 \gamma_{B}(\varphi(T))$. Hence, by Theorem 2 ,

$$
S_{B}(\varphi(T)) \leq 3 \gamma_{B}(\varphi(T)) \leq 3 \gamma_{B}\left(\varphi\left(T_{1}+T_{2}\right)\right) \leq 3 \gamma_{B}\left(T_{1}+T_{2}\right) \leq 3 K S_{B}(T) .
$$

Note that the problem with application of the above result is that $T_{1}+T_{2}$ may be much larger than $T$. We conjecture the following generalization of the above result.

Conjecture 2. Let $\varphi=\left(\varphi_{i}\right)_{i \geq 1}: T \rightarrow \ell^{2}$. If

$$
\left\|B_{\varphi(t)}-B_{\varphi(s)}\right\|_{p} \leq\left\|B_{t}-B_{s}\right\|_{p} \quad \text { for all } p \geq 2, s, t \in T
$$

then $S_{B}(\varphi(T)) \leq K S_{B}(T)$ for an absolute constant $K$.

We prove a weaker form of the conjecture. As explained before, the norm $\left\|B_{t}-B_{s}\right\|_{p}$ can be decomposed into the Gaussian and $\ell^{1}$ parts. Our condition states that if the Gaussian part of $\left\|B_{t}-B_{s}\right\|_{p}$ dominates the Gaussian part of 
$\left\|B_{\varphi(t)}-B_{\varphi(s)}\right\|_{p}$ for all $s, t \in T$ and $p \geq 1$ then $S_{B}(T)$ dominates $S_{B}(\varphi(T))$ up to an absolute constant.

TheOREM 3. Suppose that for all $s, t \in T$ and all natural $p \geq 0$ we have

$$
\inf _{\left|I^{c}\right| \leq C p} \sum_{i \in I}\left|\varphi_{i}(t)-\varphi_{i}(s)\right|^{2} \leq C^{2} \inf _{\left|I^{c}\right| \leq p} \sum_{i \in I}\left|t_{i}-s_{i}\right|^{2}
$$

for an absolute constant $C \geq 1$. Then $S_{B}(\varphi(T)) \leq K S_{B}(T)$, where $K$ is a universal constant.

REMARK 4. The result is stronger than the comparison for Bernoulli processes (14). It is easy to give an example of $\varphi$ for which the contraction on each coordinate will fail, but if for $t \in T, \varphi(t)$ is zero for all but some fixed number of coordinates then $C$ can be chosen to be appropriately large so that (16) holds for $p=0$. Consequently, the comparison will hold true. In this way Theorem 3 supports the conjecture that (15) suffices to prove that $S_{B}(\varphi(T)) \leq K S_{B}(T)$.

There is an important case for which the conjecture is true: when we assume that the supports $J(t)=\left\{i \geq 1:\left|t_{i}\right|>0\right\}$ of $t \in T$ are pairwise disjoint for all $t \in T$. It is crucial to understand that in this case the decomposition postulated in the Bernoulli Theorem can have a special form: $\pi(t)=t_{J^{1}(t)}$ and $t-\pi(t)=t_{J^{2}(t)}$, where $J^{1}(t)$ and $J^{2}(t)$ are disjoint and $J^{1}(t) \cup J^{2}(t)=J(t)$. We show this fact when proving the following result.

THEOREM 4. Suppose that 15 is satisfied and the supports $J(t)=$ $\left\{i \geq 1:\left|t_{i}\right|>0\right\}$ are pairwise disjoint for all $t \in T$. Then $S_{B}(\varphi(T)) \leq$ $K S_{B}(T)$, where $K$ is a universal constant.

As we show in the last section, results of this type are of interest when one wants to compare weak and strong moments for random series in a Banach space, as proposed by K. Oleszkiewicz in a private communication.

4. Proof of the main results. In this section we prove Theorems 3 and 4 .

Proof of Theorem 3. The main step in the proof of the Bernoulli Theorem [2, Proposition 6.2] is to show the existence of a suitable admissible sequence of partitions. Consequently, if $S_{B}(T)<\infty$ and $0 \in T$ then it is possible to define nested partitions $\mathcal{A}_{n}$ of $T$ such that $\left|\mathcal{A}_{n}\right| \leq N_{n}$. Moreover, for each $A \in \mathcal{A}_{n}$ one can find $j_{n}(A) \in \mathbb{Z}$ and $\pi_{n}(A) \in T$ (we use the notation $j_{n}(t)=j_{n}\left(A_{n}(t)\right)$ and $\pi_{n}(t)=\pi_{n}\left(A_{n}(t)\right)$, where $\left.t \in A_{n}(t) \in \mathcal{A}_{n}\right)$ which satisfy the following conditions for some $M>0$ and $r \geq 2$ :

(i) $\|t-s\|_{2} \leq \sqrt{M} r^{-j_{0}(T)}$ for $s, t \in T$;

(ii) if $n \geq 1, \mathcal{A}_{n} \ni A \subset A^{\prime} \in \mathcal{A}_{n-1}$ then either

(a) $j_{n}(A)=j_{n-1}\left(A^{\prime}\right)$ and $\pi_{n}(A)=\pi_{n-1}\left(A^{\prime}\right)$, or 
(b) $j_{n}(A)>j_{n-1}\left(A^{\prime}\right), \pi_{n}(A) \in A^{\prime}$ and

$$
\sum_{i \in I_{n}(A)} \min \left\{\left|t_{i}-\pi_{n}(A)_{i}\right|^{2}, r^{-2 j_{n}(A)}\right\} \leq M 2^{n} r^{-2 j_{n}(A)},
$$

where for any $t \in A, I_{n}(A)=I_{n}(t)=\left\{i \geq 1:\left|\pi_{k+1}(t)_{i}-\pi_{k}(t)_{i}\right| \leq\right.$ $r^{-j_{k}(t)}$ for $\left.0 \leq k \leq n-1\right\}$.

(iii) Moreover, the numbers $j_{n}(A), A \in \mathcal{A}_{n}, n \geq 0$, satisfy

$$
\sup _{t \in T} \sum_{n=0}^{\infty} 2^{n} r^{-j_{n}(t)} \leq L S_{B}(T)
$$

where $L$ is an absolute constant.

As proved in [2, Theorem 3.1], the existence of $\mathcal{A}_{n}, j_{n}(A), \pi_{n}(A), I_{n}(A)$ that satisfy conditions (i) and (ii) formulated above implies the existence of a decomposition $T_{1}, T_{2} \subset \ell^{2}$ such that $T_{1}+T_{2} \supset T$ and

$$
\sup _{t^{1} \in T_{1}}\left\|t^{1}\right\|_{1} \leq L M \sup _{t \in T} \sum_{n=0}^{\infty} 2^{n} r^{-j_{n}(t)}, \quad \gamma_{G}\left(T_{2}\right) \leq L \sqrt{M} \sup _{t \in T} \sum_{n=0}^{\infty} 2^{n} r^{-j_{n}(t)} .
$$

Together with condition (iii) we get (10). Our aim is to use the mapping $\varphi$ to transport all the required quantities to $\varphi(T)$. Before we do it, we formulate an auxiliary fact about the sets $I_{n}(A)$ : we show that we can get rid of truncation in (17) if we skip a well-controlled number of coordinates. We observe that for each $t \in A \in \mathcal{A}_{n}$ there must exist a set $J_{n}(t)$ such that $\left|J_{n}(t)^{c}\right| \leq M 2^{n+1}$ and

$$
\sum_{i \in J_{n}(t)}\left|t_{i}-\pi_{n}(t)_{i}\right|^{2} \leq M 2^{n} r^{-2 j_{n}(t)}
$$

The fact will be proved in two steps. First, we show that $\left|I_{n}(t)^{c}\right| \leq M 2^{n}$. We need only prove that $\left|I_{n}(t)\right|=\left|I_{n}\left(A_{n}(t)\right)\right| \leq 2^{n}$ if $\pi_{n-1}(t) \neq \pi_{n}(t)$, which implies $j_{n-1}(t) \neq j_{n}(t)$ and $\pi_{n}(t) \in A_{n-1}(t)$. Therefore, there exists $k \in\{1, \ldots, n\}$ such that

$$
j_{n-1}(t)=j_{n-k}(t)>j_{n-k-1}(t), \quad \text { where } \quad j_{-1}(t)=-\infty,
$$

and hence $\pi_{n}(t) \in A_{n-1}(t) \subset A_{n-k}(t)$ and $\pi_{n-1}(t)=\pi_{n-k}(t), j_{n-1}(t)=$ $j_{n-k}(t)$, so by the construction of $\left(\mathcal{A}_{n}\right)_{n \geq 0}$,

$$
\begin{aligned}
\sum_{i \in I_{n-k}(t)} & \min \left\{\left(\pi_{n}(t)_{i}-\pi_{n-1}(t)_{i}\right)^{2}, r^{-2 j_{n-1}(t)}\right\} \\
& =\sum_{i \in I_{n-k}(t)} \min \left\{\left(\pi_{n}(t)_{i}-\pi_{n-k}(t)_{i}\right)^{2}, r^{-2 j_{n-k}(t)}\right\} \leq M 2^{n-k} r^{-2 j_{n-k}(t)} .
\end{aligned}
$$

Consequently,

$$
\left|\left\{i \in I_{n-k}(t):\left|\pi_{n}(t)_{i}-\pi_{n-1}(t)_{i}\right|>r^{-j_{n-1}(t)}\right\}\right| \leq M 2^{n-k} .
$$


Obviously,

$$
\begin{aligned}
\left|I_{n}(t)^{c}\right| & \leq\left|I_{n-k}(t)^{c}\right|+\left|\left\{i \in I_{n-k}(t):\left|\pi_{n}(t)_{i}-\pi_{n-1}(t)_{i}\right|>r^{-j_{n-1}(t)}\right\}\right| \\
& \leq\left|I_{n-k}(t)^{c}\right|+M 2^{n-k} .
\end{aligned}
$$

Therefore, by induction, $\left|I_{n}(t)^{c}\right| \leq M \sum_{k=1}^{n} 2^{n-k} \leq M 2^{n}$. Now let

$$
J_{n}(t)=\left\{i \in I_{n}(A):\left|t_{i}-\pi_{n}(t)_{i}\right| \leq r^{-j_{n}(A)}\right\} .
$$

The second step is to establish that $\left|I_{n}(t) \backslash J_{n}(t)\right| \leq M 2^{n}$. Again it suffices to prove the result only for $n$ such that $j_{n}(t)>j_{n-1}(t)$. Note that by (17),

$$
\left|I_{n}(t) \backslash J_{n}(t)\right| r^{-2 j_{n}(t)}=\sum_{i \in I_{n}(A) \backslash J_{n}(t)} r^{-2 j_{n}(t)} \leq M 2^{n} r^{-2 j_{n}(t)},
$$

and hence the result holds. It remains to observe that

$$
\left|J_{n}(t)^{c}\right| \leq\left|I_{n}(t)^{c}\right|+\left|I_{n}(t) \backslash J_{n}(t)\right| \leq M\left(2^{n}+2^{n}\right) \leq M 2^{n+1} .
$$

We turn to constructing an admissible sequence of partitions together with all the related quantities for the set $\varphi(T)$. Let $\mathcal{B}_{n}$ consist of $\varphi(A)$ for $A \in \mathcal{A}_{n}$. Obviously the partitions $\mathcal{B}_{n}$ are admissible, nested and $\mathcal{B}_{0}=$ $\{\varphi(T)\}$. Moreover, for each $n \geq 0$ and $A \in \mathcal{A}_{n}$ we define

$$
\pi_{n}(\varphi(A))=\varphi\left(\pi_{n}(A)\right) \quad \text { and } \quad j_{n}(\varphi(A))=j_{n}(A),
$$

and obviously

$$
\begin{aligned}
I_{n}(\varphi(A)) & =I_{n}(\varphi(t)) \\
& =\left\{i \geq 1:\left|\varphi\left(\pi_{k+1}(t)\right)_{i}-\varphi\left(\pi_{k}(t)\right)_{i}\right| \leq r^{-j_{k}(\varphi(t))} \text { for } 0 \leq k \leq n-1\right\} .
\end{aligned}
$$

As mentioned at the beginning of this proof, in order to use [2, Theorem 3.1] we have to verify conditions (i) and (ii) for the new sequence $\mathcal{B}=\left(\mathcal{B}_{n}\right)_{n \geq 0}$ as well as $j_{n}(B), \pi_{n}(B), I_{n}(B)$ for $B \in \mathcal{B}_{n}, n \geq 0$. For this we need our main condition 16.

First, it is obvious that 16 implies for $p=0$ that

$$
\|\varphi(t)-\varphi(s)\|_{2} \leq\|t-s\|_{2} \leq \sqrt{M} r^{-j_{0}(T)} .
$$

If $A \in \mathcal{B}_{n}$ and $\varphi(A) \subset \varphi\left(A^{\prime}\right) \in \mathcal{B}_{n-1}$ then either

$$
j_{n}(\varphi(A))=j_{n}(A)=j_{n-1}\left(A^{\prime}\right)=j_{n-1}\left(\varphi\left(A^{\prime}\right)\right)
$$

and

$$
\pi_{n}(\varphi(A))=\varphi\left(\pi_{n}(A)\right)=\varphi\left(\pi_{n-1}\left(A^{\prime}\right)\right)=\pi_{n-1}\left(\varphi\left(A^{\prime}\right)\right),
$$

or $j_{n}(\varphi(A))=j_{n}(A)>j_{n-1}\left(A^{\prime}\right)=j_{n-1}\left(\varphi\left(A^{\prime}\right)\right)$. In this case we have $\pi_{n}(\varphi(A))=\varphi\left(\pi_{n}(A)\right) \in \varphi\left(A^{\prime}\right)$ and it suffices to show that

$$
\sum_{i \in I_{n}(\varphi(A))} \min \left\{\left|\varphi(t)_{i}-\varphi\left(\pi_{n}(A)\right)_{i}\right|^{2}, r^{-2 j_{n}(\varphi(A))}\right\} \leq C 2^{n} r^{-2 j_{n}(\varphi(A))} .
$$


Obviously, the problem now is that we know little about the structure of the set $I_{n}(\varphi(A))$. Therefore, we simply prove that

$$
\sum_{i \geq 1} \min \left\{\left|\varphi(t)_{i}-\varphi\left(\pi_{n}(A)\right)_{i}\right|^{2}, r^{-2 j_{n}(\varphi(A))}\right\} \leq C 2^{n} r^{-2 j_{n}(\varphi(A))} .
$$

Clearly,

$$
\begin{aligned}
& \sum_{i \geq 1} \min \left\{\left|\varphi(t)_{i}-\varphi\left(\pi_{n}(A)\right)_{i}\right|^{2}, r^{-2 j_{n}(\varphi(A))}\right\} \\
& \quad \leq C_{2} 2^{n} r^{-2 j_{n}(A)}+\inf _{\left|I^{c}\right| \leq C_{2} 2^{n}} \sum_{i \in I}\left|\varphi(t)_{i}-\varphi\left(\pi_{n}(A)\right)_{i}\right|^{2} .
\end{aligned}
$$

We can choose $C_{2} \geq 2 C M$ in such a way that by 16 we get

$$
\begin{aligned}
\inf _{\left|I^{c}\right| \leq C_{2} 2^{n}} \sum_{i \in I} \mid \varphi(t)_{i}- & \left.\varphi\left(\pi_{n}(A)\right)_{i}\right|^{2} \\
& \leq C^{2} \inf _{\left|I^{c}\right| \leq M 2^{n+1}} \sum_{i \in I}\left|t_{i}-\pi_{n}(A)_{i}\right|^{2} \\
& \leq C^{2} \sum_{i \in J_{n}(t)}\left|t_{i}-\pi_{n}(A)_{i}\right|^{2} .
\end{aligned}
$$

Hence, by (19) and 21),

$$
\sum_{i \geq 1} \min \left\{\left|\varphi(t)_{i}-\varphi\left(\pi_{n}(A)\right)_{i}\right|^{2}, r^{-2 j_{n}(\varphi(A))}\right\} \leq\left(C_{2}+C^{2} M\right) 2^{n} r^{-2 j_{n}(A)},
$$

which proves 20 with $C_{3}=C_{2}+C^{2} M$.

We have proved that the assumptions required in [2, Theorem 3.1] are satisfied for $\left(\mathcal{B}_{n}\right)_{n \geq 0}$ and the related quantities. Consequently, there exists a decomposition $S_{1}, S_{2} \subset \ell^{2}$ such that $S_{1}+S_{2} \supset \varphi(T)$ and

$$
\sup _{s \in S_{1}}\|s\|_{1} \leq L C \sup _{t \in \varphi(T)} \sum_{n \geq 0} 2^{n} r^{-j_{n}(t)}, \gamma_{G}\left(S_{2}\right) \leq L \sqrt{C} \sup _{t \in \varphi(T)} \sum_{n \geq 0} 2^{n} r^{-j_{n}(t)} .
$$

Since $j_{n}(\varphi(t))=j_{n}(t)$ and we have 18$)$ for $\left(\mathcal{A}_{n}\right)_{n \geq 0}$, we obtain

$$
\sup _{t \in \varphi(T)} \sum_{n \geq 0} 2^{n} r^{-j_{n}(t)} \leq L S_{B}(T) .
$$

This implies that

$$
S_{B}(\varphi(T)) \leq S_{B}\left(S_{1}\right)+S_{B}\left(S_{2}\right) \leq K S_{B}(T)
$$

for a universal constant $K$ and ends the proof.

The second case we consider is when the supports $J(t)=\left\{i \geq 1:\left|t_{i}\right|>0\right\}$ are pairwise disjoint for all $t \in T$. The proof requires the following notation. For any $t \in \ell^{2}$ and $J \subset\{1,2, \ldots\}$ we define $t 1_{J} \in \ell^{2}$ such that $\left(t 1_{J}\right)_{i}=t_{i}$ for $i \in J$ and $\left(t 1_{J}\right)_{i}=0$ otherwise. 
Proof of Theorem 4. Obviously, we may require that $S_{B}(T)<\infty$. We additionally assume that $0 \in T$. This simplifies the proof, but the proof works also for the general case as we will point out at the end.

Recall that by the Bernoulli Theorem [2] there exists a decomposition $T_{1}+T_{2} \supset T$ such that

$$
S_{B}(T) \geq K^{-1}\left(\sup _{t \in T_{1}}\|t\|_{1}+\gamma_{G}\left(T_{2}\right)\right)
$$

where $K$ is an absolute constant. Obviously, we may think of $K$ as suitably large. We can represent the decomposition by $\pi: T \rightarrow \ell^{2}$ such that $T_{2}=$ $\{\pi(t): t \in T\}$ and $T_{1}=\{t-\pi(t): t \in T\}$. We show that under the disjoint supports assumption we may additionally require that $\pi(t)=t 1_{J^{2}(t)}$ and $t-\pi(t)=t 1_{J^{1}(t)}$ where $J^{1}(t)$ and $J^{2}(t)$ are disjoint subsets of $J(t)$ such that $J^{1}(t) \cup J^{2}(t)=J(t)$. Moreover, $J^{2}(t)=\left\{i \in J(t):\left|t_{i}\right| \leq p(t)\right\}$ for some suitably chosen $p(t) \geq 0$.

In order to prove the result we have to look closer into the definition of $\pi(t)$ in [2, proof of Theorem 3.1]. The definition is based on the construction of admissible sequences of partitions we have described in the proof of Theorem 3 above. Using the notation introduced there, let

$$
m(t, i)=\inf \left\{n \geq 0:\left|\pi_{n+1}(t)_{i}-\pi_{n}(t)_{i}\right|>r^{-j_{n}(t)}\right\}, \quad t \in T, i \geq 1 .
$$

Note that $S_{B}(T)$ is comparable with $\sup _{t \in T} \sum_{n \geq 0} 2^{n} r^{-j_{n}(t)}$. Therefore, if $S_{B}(T)$ is finite then necessarily $\lim _{n \rightarrow \infty} j_{n}(t)=\infty$ for all $t \in T$. From the partition construction in [2, Section 6] we know that we can additionally assume a regularity condition on $j_{n}(t), n \geq 0$, namely

$$
j_{n}(t) \leq j_{n-1}(t)+2 \quad \text { for all } n \geq 0,
$$

and for technical purposes we take $j_{-1}(t)=-\infty$. As in [2, proof of Theorem 3.1] the Bernoulli decomposition $\pi(t)$ is given by $\pi(t)_{i}=\pi_{m(t, i)}(t)_{i}$, where if $m(t, i)=\infty$ the definition means that $\pi(t)_{i}=\lim _{n \rightarrow \infty} \pi_{n}(t)_{i}$ and the limit exists. Consequently, denoting $J_{n}(t)=\{i \geq 1: m(t, i)=n\}$ and $J_{\infty}(t)=\{i \geq 1: m(t, i)=\infty\}$ we get

$$
\pi(t)=\sum_{n \geq 0} \pi_{n}(t) 1_{J_{n}(t)}+\pi(t) 1_{J_{\infty}(t)} .
$$

Clearly, $J_{n}(t), n \geq 0$, and $J_{\infty}(t)$ are disjoint. Note also that if $m(t, i)=\infty$ and $i \in J(\pi(t))$, then there must exist $n \geq 0$ such that $\left|\pi_{k}(t)_{i}\right|>0$ for all $k \geq n$. Due to the disjoint supports assumption this is only possible if there exists $n \geq 0$ such that $\pi_{n}(t)_{i}=\pi_{n+1}(t)_{i}=\cdots$. Now, if there exists $m \geq 0$ such that $A_{m}(t)=\{t\}$ we define

$$
\tau(t)=\inf \left\{n \geq 0: A_{n}(t)=\{t\}=\left\{\pi_{n}(t)\right\}, j_{n-1}(t)<j_{n}(t)\right\},
$$

otherwise $\tau(t)=\infty$. The time $\tau(t)$ is of special nature in the sense that without loss of generality we may assume that $j_{n}(t)=j_{n-1}(t)+2$ for $n \geq \tau(t)$. 
This is due to the fact that partitioning ceases after that time. Now, we define

$$
J^{2}(t)=\left\{i \in J(t):\left|t_{i}\right| \leq r^{-j_{\tau(t)}(t)-1}\right\}, \quad J^{1}(t)=J(t) \backslash J^{2}(t) .
$$

We can now introduce an improved version of $\pi$ denoted by $\bar{\pi}$ and given by

$$
\bar{\pi}(t)=t 1_{J^{2}(t)} .
$$

It is clear that

$$
\|t-\bar{\pi}(t)\|_{1}=\left\|t 1_{J^{1}(t)}\right\|_{1}
$$

For $n \geq 0$ let

$$
L_{n}(t)=\left\{i \in J(t): r^{-j_{n}(t)}<\left|t_{i}\right| \leq r^{-j_{n-1}(t)}\right\} .
$$

Observe that $J^{1}(t)=\bigcup_{n<\tau(t)} L_{n}(t)$. If $i \in L_{n}(t), n \geq 0$, then we may find $0 \leq m \leq n$ such that $j_{m-1}(t)<j_{m}(t)=j_{m+1}(t)=\cdots=j_{n}(t)$. Consequently, by the definition (17) of $I_{n}(t)$, for all $s \in A_{m}(t)$,

$$
\begin{array}{r}
\sum_{i \in I_{n}(t)} \min \left\{\left|s_{i}-\pi_{n}(t)_{i}\right|^{2}, r^{-2 j_{n}(t)}\right\}=\sum_{i \in I_{m}(t)} \min \left\{\left|s_{i}-\pi_{m}(t)_{i}\right|^{2}, r^{-2 j_{m}(t)}\right\} \\
\leq M 2^{m} r^{-2 j_{m}(t)}=M 2^{m} r^{-2 j_{n}(t)} \leq M 2^{n} r^{-2 j_{n}(t)} .
\end{array}
$$

We need to show that the decomposition $\bar{\pi}$ is of the right form, i.e. it satisfies $(22)$. To this end we need to investigate a few cases according to different possible paths of approximations $\pi$. First suppose that $t \neq \pi_{n}(t)$. Then we may use the above inequality for $s=t$ and thanks to disjoint supports we have

$$
\left|I_{n}(t) \cap L_{n}(t)\right| r^{-2 j_{n}(t)} \leq \sum_{i \in I_{n}(t)} \min \left\{\left|s_{i}-\pi_{n}(t)\right|_{i}^{2}, r^{-2 j_{n}(t)}\right\} \leq M 2^{n} r^{-2 j_{n}(t)},
$$

so $\left|I_{n}(t) \cap L_{n}(t)\right| \leq M 2^{n}$. The same inequality holds if $t=\pi_{n}(t)$ but $A_{m}(t) \neq\{t\}$.

We show that $L_{n}(t) \subset I_{n}(t)$. Indeed, suppose that $i \notin I_{n}(t)$. This means that for some $k \in\{0,1, \ldots, n-1\}$ we have $\left|\pi_{k+1}(t)_{i}-\pi_{k}(t)_{i}\right|>r^{-j_{k}(t)}$. This may concern $i \in J(t)$ only if $\pi_{k+1}(t)=t, \pi_{k}(t) \neq t$ or $\pi_{k}(t)=t$ and $\pi_{k+1}(t) \neq t$, but then it means that $\left|t_{i}\right|>r^{-j_{k}(t)} \geq r^{-j_{n-1}(t)}$, i.e. $i \notin L_{n}(t)$, as desired.

For $1 \leq n<\tau(t)$ this implies that

$$
\sum_{i \in L_{n}(t)}\left|t_{i}\right| \leq M 2^{n} r^{-j_{n-1}(t)}
$$

For $n=0$ we use simply $\left|t_{i}\right| \leq 2 S_{B}(T)$ and hence

$$
\sum_{i \in L_{0}(t)}\left|t_{i}\right| \leq 2 M S_{B}(T) .
$$


Now suppose that $t=\pi_{n}(t)=\pi_{m}(t)$ and $A_{m}(t)=\{t\}$. If either $t \neq \pi_{m-1}(t)$ or $\{t\} \neq A_{m-1}(t)$, then $\tau(t)=m$; otherwise $\tau(t)<m$. If $\tau(t)=m$, then by the above argument,

$$
\sum_{i \in L_{n}(t)}\left|t_{i}\right|^{2}=\sum_{i \in L_{n}(t)} \min \left\{\left|t_{i}\right|^{2}, r^{-2 j_{m-1}(t)}\right\} \leq M 2^{m-1} r^{-2 j_{m-1}(t)},
$$

and since $\left|t_{i}\right| \geq r^{-j_{m}(t)-1}$ and $j_{m}(t)=j_{m-1}(t)+2$, we have

$$
\sum_{i \in L_{n}(t)}\left|t_{i}\right| \leq M 2^{m-1} r^{-2 j_{m-1}(t)+j_{m}(t)} \leq M 2^{m-1} r^{-j_{m-1}(t)+2} .
$$

We have the remaining bound

$$
\sum_{i \in L_{n}(t)}\left|t_{i}\right| \leq M 2^{\tau(t)-1} r^{-j_{\tau(t)-1}(t)+2} .
$$

Combining (24)-26) we conclude by 18 that

$$
\begin{aligned}
\left\|t 1_{J^{1}(t)}\right\|_{1} & \leq 2 M S_{B}(T)+2 M \sum_{n=0}^{\tau(t)-2} r^{-j_{n}(t)} 2^{n}+M 2^{\tau(t)-1} r^{-j_{\tau(t)-1}(t)+2} \\
& \leq 2 M L S_{B}(T)
\end{aligned}
$$

where $L$ is an absolute constant.

Now consider $s, t \in T, s \neq t$. In order to prove that

$$
\|\bar{\pi}(s)-\bar{\pi}(t)\|_{2}=\left\|t 1_{J^{2}(t)}-s 1_{J^{2}(s)}\right\|_{2} \leq\|\pi(t)-\pi(s)\|_{2}
$$

we have to argue that $J^{2}(t) \cap J(\pi(s))=\emptyset, J^{2}(s) \cap J(\pi(t))=\emptyset$. Note that $J^{2}(t) \subset J_{\infty}(t)$ and $J^{2}(t) \subset J_{\infty}(s)$. Moreover, $J_{\infty}(s)$ and $J_{\infty}(s)$ are disjoint. Obviously, it suffices to show that $J^{2}(t) \cap J(\pi(s))=\emptyset$.

First, note that $J^{2}(t) \cap J_{\infty}(s)=\emptyset$. Indeed, if this set were non-empty then for a given $n \geq 0$ we would have $t=\pi_{n}(s)=\pi_{n+1}(s)=\cdots$, but then $s \in A_{n}(t)$ for all $n \geq 0$ and therefore $\tau(t)=\infty$. This would imply $J^{2}(t)=\emptyset$, a contradiction.

Suppose that $i \in J^{2}(t)$ and $i \in J_{n}(s)$. This is only possible if $\pi_{n}(s)=t$ and $\pi_{n+1}(s) \neq \pi_{n}(s)=t$ and $r^{-j_{n}(s)}<\left|\pi_{n}(s)_{i}\right|$. Let $m \geq 0$ be such that $j_{m-1}(s)<j_{m}(s)=j_{m+1}(s)=\cdots=j_{n}(s)$. Then either $m=0$, or $m \geq 1$ and $t=\pi_{n}(s)=\pi_{m}(s) \in A_{m-1}(s)$, which means that $A_{m-1}(s)=A_{m-1}(t)$ and $j_{m-1}(s)=j_{m-1}(t)$. Therefore, $\tau(t) \geq m$ and $j_{\tau(t)}(t)>j_{m-1}(t)$. As $i \in J^{2}(t) \cap J_{n}(s)$, we have

$$
\begin{aligned}
r^{-j_{m-1}(t)-2} & =r^{-j_{m-1}(s)-2} \leq r^{-j_{m}(s)} \\
& <\left|t_{i}\right| \leq r^{-j_{\tau(t)}(t)}=r^{-j_{\tau(t)-1}(t)-2} \leq r^{-j_{m-1}(t)-2},
\end{aligned}
$$

a contradiction. If $m=0$, then the argument is trivial. 
Summing up, by (27) we have

$$
\sup _{t \in T}\|t-\bar{\pi}(t)\|_{1} \leq L S_{B}(T)
$$

and by (28) and Gaussian comparison we have $\gamma_{G}(\pi(T)) \leq \gamma_{G}(\bar{\pi}(T))$, which means that our improved version of $\pi$ satisfies

$$
S_{B}(T) \geq K^{-1}\left(\sup _{t \in T}\|t-\bar{\pi}(t)\|_{1}+\gamma_{G}(\bar{\pi}(T))\right)
$$

where $K$ is a universal constant. In this way we have proved that we may additionally require that $\pi(t)=t 1_{J^{2}(t)}$ and $t-\pi(t)=t 1_{J^{1}(t)}$ for some disjoint $J^{1}(t), J^{2}(t)$ such that $J^{1}(t) \cup J^{2}(t)=J(t)$. Recall that $J^{2}(t)$ in each case is of the form $\left\{i \in J(t):\left|t_{i}\right| \leq r(t)\right\}$ for a given $r(t) \geq 0$.

We turn to the main part of the proof. Let $p(t)$ be the smallest positive integer such that

$$
\sqrt{p(t)}\left\|t 1_{J^{2}(t)}\right\|_{2} \geq K S_{B}(T) \geq\left\|t 1_{J^{1}(t)}\right\|_{1} .
$$

Note that it is possible that $J^{2}(t)=\emptyset$, in which case we may think of $p(t)$ as equal to $\infty$. Since $K$ is large enough and $S_{B}(T) \geq \frac{1}{2} \sup _{t \in T}\|t\|_{2}$, it is clear that $p(t)$ must be at least, say, 2 . Consequently, by the choice of $p(t)$,

$$
\sqrt{p(t)}\left\|t 1_{J^{2}(t)}\right\|_{2} \leq 2 K S_{B}(T) .
$$

The last step is to define a suitable decomposition for $\varphi(T)$. For each $t \in T$ we define $\pi(\varphi(t))=t_{J^{2}(\varphi(t))}$ and $\varphi(t)-\pi(\varphi(t))=t_{J^{1}(\varphi(t))}$, where $J^{2}(\varphi(t))$ and $J^{1}(\varphi(t))$ are defined by the decomposition of the norm $\left\|B_{\varphi(t)}\right\|_{p(t)}$, i.e.

$$
\sum_{i \in J^{1}(\varphi(t))}\left|\varphi(t)_{i}\right|=\sup _{\left|I^{c}\right| \leq p(t)} \sum_{i \in I^{c}}\left|\varphi(t)_{i}\right|
$$

and

$$
\sum_{i \in J^{2}(\varphi(t))}\left|\varphi(t)_{i}\right|^{2}=\inf _{\left|I^{c}\right| \leq p(t)} \sum_{i \in I}\left|\varphi(t)_{i}\right|^{2} .
$$

Consequently, by the decomposition (8) and the main assumption (15),

$$
\begin{aligned}
& \sum_{i \in J^{1}(\varphi(t))}\left|\varphi(t)_{i}\right|+\sqrt{p(t)}\left(\sum_{i \in J^{2}(\varphi(t))}\left|\varphi(t)_{i}\right|^{2}\right)^{1 / 2} \\
& \leq 4\left\|B_{\varphi(t)}\right\|_{p(t)} \leq 4\left\|B_{t}\right\|_{p(t)} \leq 4\left(\left\|t 1_{J^{1}(t)}\right\|_{1}+\sqrt{p(t)}\left\|t 1_{J^{2}(t)}\right\|_{2}\right) .
\end{aligned}
$$

Therefore, by (29), (30),

$$
\sum_{i \in J^{1}(\varphi(t))}\left|\varphi(t)_{i}\right| \leq K_{1} S_{B}(T) .
$$


Moreover, by (29),

$$
\left(\sum_{i \in J^{2}(\varphi(t))}\left|\varphi(t)_{i}\right|^{2}\right)^{1 / 2} \leq K_{2}\left\|t 1_{J^{2}(t)}\right\|_{2} .
$$

This implies that

$$
\begin{aligned}
\|\pi(\varphi(t))-\pi(\varphi(s))\|_{2} & \leq\|\pi(\varphi(t))\|_{2}+\|\pi(\varphi(s))\|_{2} \\
& \leq K_{2}\left(\left\|t 1_{J^{2}(t)}\right\|_{2}+\left\|s 1_{J^{2}(s)}\right\|_{2}\right) \leq K_{3}\|\pi(t)-\pi(s)\|_{2} .
\end{aligned}
$$

Therefore, by Gaussian comparison, we get $\gamma_{G}(\pi(\varphi(T))) \leq K \gamma_{G}(\pi(T))$ and hence finally

$$
S_{B}(\varphi(T)) \leq K\left(\sup _{t \in T}\|\pi(\varphi(t))\|_{1}+\gamma_{G}(\pi(\varphi(T)))\right) \leq K L S_{B}(T) .
$$

This ends the proof in the case when $0 \in T$. For the general case the proof follows the same lines, where instead of $t$ we consider $t-\pi_{0}(t)$. Notice that formally this may not obey the disjoint supports assumption, but it does not qualitatively affect the argument presented above.

Note that the above proof works since in the case of disjoint supports we have almost perfect knowledge about the decomposition in the Bernoulli Theorem. On the other hand, it is not difficult to give an alternative proof based on the independence of the variables $B_{t}, t \in T$, but it is worth seeing what the decomposition in [2, Theorem 3.1] should be in order to make Bernoulli comparison possible.

5. The Oleszkiewicz problem. In this section we apply our result to compare expectations of norms of random series in a Banach space. First, we prove a general result which concerns $\varphi: T \rightarrow \ell^{2}$ where $\varphi$ is linear, $T$ is convex and $T=-T$. Then the assumption 12 becomes

$$
\left\|B_{\varphi(u)}\right\|_{p} \leq C\left\|B_{u}\right\|_{p} \quad \text { for all } p \geq 1 \text { and } u \in \operatorname{cl}(\operatorname{Lin}(T)),
$$

where $\operatorname{Lin}(T)$ is the linear space spanned by the set $T$. This is because by the assumptions on $T$ any point $u \in \operatorname{Lin}(T)$ can be represented as $c \cdot t$, where $c \in \mathbb{R}$ and $t \in T$. By the linearity of $\varphi$,

$$
\left\|B_{\varphi(u)}\right\|_{p}=|c|\left\|B_{\varphi(t)}\right\|_{p} \leq C|c|\left\|B_{t}\right\|_{p}=C\left\|B_{u}\right\|_{p} .
$$

On the other hand, we can easily extend the condition (31) to the closure of $\operatorname{Lin}(T)$.

We turn to proving that if $\operatorname{cl}(\operatorname{Lin}(T))=\ell^{2}$ then 31 implies that $S_{B}(T)$ dominates $S_{B}(\varphi(T))$.

Theorem 5. Suppose that $T=-T, T$ is convex and $\operatorname{cl}(\operatorname{Lin}(T))=\ell^{2}$. If $\varphi$ is linear and satisfies (12) then $S_{B}(\varphi(T)) \leq K S_{B}(T)$, where $K$ is a universal constant. 
Proof. By the Bernoulli Theorem [2] there exist $T_{1}, T_{2}$ such that $T \subset$ $T_{1}+T_{2}$ and

$$
S_{B}(T) \geq L^{-1}\left(\sup _{t \in T_{1}}\|t\|_{1}+\gamma_{G}\left(T_{2}\right)\right) .
$$

Since $\varphi$ is linear, it can be easily extended to $\operatorname{cl}(\operatorname{Lin}(T))=\ell^{2}$ and thus we can define $S_{i}=\varphi\left(T_{i}\right), i \in\{1,2\}$. Obviously $S_{1}+S_{2} \supset \varphi(T)$; moreover, 31 implies in particular that

$$
\|\varphi(u)\|_{1}=\left\|B_{\varphi(u)}\right\|_{\infty} \leq C\left\|B_{u}\right\|_{\infty}=C\|u\|_{1}
$$

and

$$
\|\varphi(u)-\varphi(v)\|_{2}=\left\|B_{\varphi(u-v)}\right\|_{2} \leq C\left\|B_{u-v}\right\|_{2}=C\|u-v\|_{2} .
$$

Consequently,

$$
\sup _{s \in S_{1}}\|s\|_{1}=\sup _{t \in T_{1}}\|\varphi(t)\|_{1} \leq C \sup _{t \in T_{1}}\|t\|_{1}
$$

and

$$
\gamma_{G}\left(S_{2}\right)=\gamma_{G}\left(\varphi\left(T_{2}\right)\right) \leq C \gamma_{G}\left(T_{2}\right)
$$

Therefore

$$
\begin{aligned}
S_{B}(\varphi(T)) & \leq S_{B}\left(S_{1}\right)+S_{B}\left(S_{2}\right) \leq K\left(\sup _{s \in S_{1}}\|s\|_{1}+\gamma_{G}\left(S_{2}\right)\right) \\
& \leq C K\left(\sup _{t \in T_{1}}\|t\|_{1}+\gamma_{G}\left(T_{2}\right)\right) \leq C K^{2} S_{B}(T) .
\end{aligned}
$$

We aim to study the question, posed by Oleszkiewicz, of comparability of weak and strong moments for Bernoulli series in a Banach space. Let $x_{i}, y_{i}$, $i \geq 1$, be vectors in a Banach space $(B,\|\cdot\|)$. Suppose that for all $x^{*} \in B^{*}$ and $u \geq 0$,

$$
\mathbf{P}\left(\left|\sum_{i \geq 1} x^{*}\left(x_{i}\right) \varepsilon_{i}\right|>u\right) \leq \bar{C} \mathbf{P}\left(\left|\sum_{i \geq 1} x^{*}\left(y_{i}\right) \varepsilon_{i}\right|>\bar{C}^{-1} u\right) .
$$

This property is called weak tail domination. As explained in the introduction, weak tail domination can be understood in terms of comparability of weak moments, i.e. for any integer $p \geq 1$ and $x^{*} \in B^{*}$,

$$
\left\|\sum_{i \geq 1} x^{*}\left(x_{i}\right) \varepsilon_{i}\right\|_{p} \leq C\left\|\sum_{i \geq 1} x^{*}\left(y_{i}\right) \varepsilon_{i}\right\|_{p}
$$

Oleszkiewicz asked whether or not this implies comparability of strong moments, that is, whether (32) or rather (33) implies that

$$
\begin{aligned}
\mathbf{E}\left\|\sum_{i \geq 1} x_{i} \varepsilon_{i}\right\| & =\mathbf{E} \sup _{x^{*} \in B_{1}^{*}} \sum_{i \geq 1} x^{*}\left(x_{i}\right) \varepsilon_{i} \\
& \leq K \mathbf{E} \sup _{x^{*} \in B_{1}^{*}} \sum_{i \geq 1} x^{*}\left(y_{i}\right) \varepsilon_{i}=K \mathbf{E}\left\|\sum_{i \geq 1} y_{i} \varepsilon_{i}\right\|,
\end{aligned}
$$


where $K$ is an absolute constant. Note that in the Oleszkiewicz problem one may assume that $B$ is a separable space since we can easily restrict the argument to the closure of $\operatorname{Lin}\left(y_{1}, x_{1}, y_{2}, x_{2}, \ldots\right)$. Therefore

$$
\mathbf{E}\left\|\sum_{i \geq 1} y_{i} \varepsilon_{i}\right\|=\sup _{F \subset B_{1}^{*}} \mathbf{E} \sup _{x^{*} \in F}\left|\sum_{i \geq 1} x^{*}\left(y_{i}\right) \varepsilon_{i}\right|,
$$

where $F$ runs through all finite sets contained in $B_{1}^{*}=\left\{x^{*} \in B^{*}:\left\|x^{*}\right\| \leq 1\right\}$. We may assume that $\mathbf{E}\left\|\sum_{i \geq 1} y_{i} \varepsilon_{i}\right\|<\infty$ since otherwise there is nothing to prove. Consequently, for each $x^{*} \in B^{*}$ the series $\sum_{i \geq 1} x^{*}\left(y_{i}\right) \varepsilon_{i}$ is convergent, which is equivalent to $\sum_{i \geq 1}\left(x^{*}\left(y_{i}\right)\right)^{2}<\infty$. Let $Q: B^{*} \rightarrow \ell^{2}$ be defined by $Q\left(x^{*}\right)=\left(x^{*}\left(y_{i}\right)\right)_{i \geq 1}$. It is clear that $Q: B^{*} / \operatorname{ker} Q \rightarrow \ell^{2}$ is a linear isomorphism onto a closed linear subspace of $\ell^{2}$. We apply Theorem 5 to get the following result.

Corollary 3. Suppose that $Q$ is onto $\ell^{2}$. Then (32) implies (34).

Unfortunately, if $Q$ is not onto $\ell^{2}$ then the above argument fails. Still it is believed that the comparison holds. A partial result can be deduced from Theorem 3:

Corollary 4. Suppose that for each $x^{*} \in B^{*}$ and $p \geq 0$,

$$
\inf _{\left|I^{c}\right| \leq C p} \sum_{i \in I}\left|x^{*}\left(x_{i}\right)\right|^{2} \leq C^{2} \inf _{\left|I^{c}\right| \leq p} \sum_{i \in I}\left|x^{*}\left(y_{i}\right)\right|^{2} .
$$

Then (34) holds, i.e.

$$
\mathbf{E}\left\|\sum_{i \geq 1} x_{i} \varepsilon_{i}\right\| \leq K \mathbf{E}\left\|\sum_{i \geq 1} y_{i} \varepsilon_{i}\right\|
$$

Proof. It suffices to notice that (35) implies (16) and then apply Theorem 3 ,

Acknowledgements. We would like to thank the referee for his comments on the paper which allowed us to improve Theorems 1 and 2 .

This research was partially supported by National Science Centre, Poland, grant 2015/18/A/ST1/00553.

\section{References}

[1] W. Beckner, Inequalities in Fourier analysis, Ann. of Math. 102 (1975), 159-182.

[2] W. Bednorz and R. Latała, On the boundedness of Bernoulli processes, Ann. of Math. 180 (2014), 1167-1203.

[3] X. Fernique, Régularité des trajectoires des fonctions aléatoires gaussiennes, in: École d'Été de Probabilités de Saint-Flour, IV-1974, Lecture Notes in Math. 480, Springer, Berlin, 1975, 1-96.

[4] D. J. H. Garling, Inequalities: A Journey into Linear Analysis, Cambridge Univ. Press, Cambridge, 2007. 
[5] P. Hitczenko, Domination inequality for martingale transforms of a Rademacher sequence, Israel J. Math. 84 (1993), 161-178.

[6] P. Hitczenko and S. Kwapien, On the Rademacher series, in: J. Hoffmann-Jørgensen et al. (eds.), Probability in Banach Spaces, 9, Progr. Probab. 35, Birkhäuser Boston, Boston, MA, 1994, 31-36.

[7] S. Kwapień and W. A. Woyczyński, Random Series and Stochastic Integrals: Single and Multiple, Birkhäuser Boston, Boston, MA, 1992.

[8] R. Latała, Sudakov minoration principle and supremum of some processes, Geom. Funct. Anal. 7 (1997), 936-953.

[9] R. Latała, Moments of unconditional logarithmically concave vectors, in: Geometric Aspects of Functional Analysis, Israel Seminar 2006-2010, Lecture Notes in Math. 2050, Springer, 2012, 301-315.

[10] R. Latała and T. Tkocz, A note on suprema of canonical processes based on random variables with regular moments, Electron. J. Probab. 2015, no. 20, 17 pp.

[11] M. Ledoux and M. Talagrand, Probability in Banach Spaces. Isoperimetry and Processes, Ergeb. Math. Grenzgeb. 23, Springer, Berlin, 1991.

[12] M. B. Marcus and J. Rosen, Markov Processes, Gaussian Processes, and Local Times, Cambridge Stud. Adv. Math. 100, Cambridge Univ. Press, Cambridge, 2006.

[13] S. Mendelson and G. Paouris, On generic chaining and the smallest singular value of random matrices with heavy tails, J. Funct. Anal. 262 (2012), 3775-3811.

[14] S. Montgomery-Smith, Comparison of sums of independent identically distributed random vectors, Probab. Math. Statist. 14 (1992), 281-285.

[15] G. Pisier, Conditions d'entropie assurant la continuité de certains processus et applications à l'analyse harmonique, in: Séminaire d'Analyse Fonctionnelle 1979-1980, exp. 13-14, École Polytech., Palaiseau, 43 pp.

[16] M. Talagrand, Regularity of Gaussian processes, Acta Math. 159 (1987), 99-149.

[17] M. Talagrand, The supremum of some canonical processes, Amer. J. Math. 116 (1994), $283-325$.

[18] M. Talagrand, Regularity of infinitely divisible processes, Ann. Probab. 20 (1993), $362-432$.

[19] M. Talagrand, Upper and Lower Bounds for Stochastic Processes. Modern Methods and Classical Problems, Ergeb. Math. Grenzgeb. 60, Springer, New York, 2014.

Witold Bednorz, Rafał Martynek

Institute of Mathematics

University of Warsaw

Banacha 2

02-097 Warszawa, Poland

E-mail: wbednorz@mimuw.edu.pl

r.martynek@mimuw.edu.pl 
